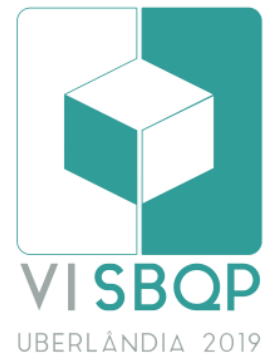

\title{
FABRICAÇÃO DIGITAL, RESÍDUOS E MEIO AMBIENTE: O CASO DA CNC FRESADORA
}

\author{
ABRÃO, Júlia Souza \\ UFU, e-mail: juliaabraoufu@gmail.com \\ NUNES, Viviane dos Guimarães Alvim
}

UFU, e-mail: viviane.nunes@ufu.br

\begin{abstract}
RESUMO
A discussão proposta neste artigo visa contribuir com questões relacionadas à inserção da Fabricação Digital nos processos produtivos e os impactos ambientais ocasionados pelo volume de material descartado durante o processo de fabricação das manufaturas subtrativas (CNC fresadora). O artigo é estruturado a partir de estudos de uma pesquisa de mestrado do Programa de Pós-Graduação em Arquitetura e Urbanismo (PPGAU - UFU) intitulada: "Projetar antevendo a fabricação: Estratégias eco eficientes para designers em processos de fabricação digital subtrativa". A metodologia aplicada na pesquisa foi revisão de literatura, abordando conceitos relacionados à Fabricação Digital (KOLAREVIC, 2005; OOSTERHUIS, 2005; OXMAN, 2006; BARBOSA NETO et al., 2014), Processo de projeto (Hopkinson et al, 2006; PUPO, 2009) e Sustentabilidade (MANZINI, 2008; GERSHENFELD, 2012; FLORES; TERRIBILE, 2015). Tendo em vista os diversos impactos causados pela produção em geral e, mais especificamente, daquela resultante de projetos desarticulados dos processos produtivos e os materiais utilizados, este artigo aponta que a ética associada ao conhecimento de estratégias projetuais são aspectos fundamentais para se reduzir o impacto ambiental provocado pelo uso equivocado das manufaturas digitais e, mais importante, para se promover um modelo de produção mais sustentável.
\end{abstract}

Palavras-chave: Fabricação Digital; Processo de projeto; Sustentabilidade no Design; Ética Projetual

\begin{abstract}
The subtractive manufactures of CNC (milling machine). The article is structured from studies of a master's research of the Post-Graduate Program in Architecture and Urbanism (PPGAU - UFU) entitled "Designing foreseeing a manufacturing: Eco-efficient strategies for designing in subtractive digital manufacturing processes". In this paper, we present the results of the literature on the design of the Digital Fabrication (KOLAREVIC, 2005; OOSTERHUIS, 2005; OXMAN, 2006; BARBOSA NETO et al., 2014. 2009) and Sustainability (MANZINI, 2008, GERSHENFELD, 2012, FLORES, TERRIBILE, 2015). The aim to the level of the production in the general and, more born, the off project of disarticulated the processes product and the materials used, this article that has the right related to knowledge of perspectives projects are essential aspects to the amount the environmental impact caused by the misuse of digital manufactures and, more importantly, to promote a more sustainable production model.
\end{abstract}

Keywords: Digital Manufacturing; Project process; Sustainability of Design; Project Ethics

\section{INTRODUÇÃO}

Nas últimas décadas, o avanço tecnológico digital alterou a configuração de trabalho, adquirindo a possibilidade de atuar em novos campos, incorporando o caráter interdisciplinar em sua utilização (MENICHINELLI, 2017).

ABRÃO, J. S.; NUNES, V. G. A. Fabricação Digital, Resíduos e Meio Ambiente: o Caso da CNC Fresadora. In: SIMPÓSIO BRASILEIRO DE QUALIDADE DO PROJETO NO AMBIENTE CONSTRUÍDO, $6 .$, 2019, Uberlândia. Anais... Uberlândia: PPGAU/FAUED/UFU, 2019. p. 107-114. DOI https://doi.org/10.14393/sbqp19011. 
Com o surgimento da Fabricação Digital (FD), a partir 1980, teve início uma nova configuração no processo de fabricação, onde as máquinas, aliadas aos novos campos computacionais, passaram a ser controladas por computadores, tecnologia essa entendida a partir da sigla CNC (Controle Numérico Computadorizado) (KOLAREVIC, 2005).

Essa nova tecnologia, juntamente com os novos softwares de modelagem, possibilitou, assim, a criação de peças tanto bidimensionais quanto tridimensionais e também disponibilizou uma série de ferramentas para a execução de projetos. Anteriormente ao surgimento das tecnologias digitais, os designers eram disciplinados a criar projetos com geometrias simples, no sentido de viabilizar a fabricação das peças com mais facilidade (Hopkinson et al, 2006).

Em contraposição aos modelos anteriores, a fabricação digital possui como principal característica a possibilidade de gerar formas complexas, configurando, por sua vez, um novo modelo de elaboração de projetos, que permite uma estética diferenciada e ampla variedade de geometrias em sua composição.

Segundo Kolarevic (2005), esses meios de fabricação digital são definidos por tipologias, dentre elas: Fabricação Subtrativa, Fabricação Aditiva e Fabricação Formativa, contando com a grande diversidade de máquinas. A pesquisa em questão dá ênfase à máquina CNC Fresadora Router, de tipologia subtrativa, utilizada para o fresamento de formas bidimensionais em materiais como madeira, MDF, entre outros. $O$ enfoque dado à essa tipologia decorre das preocupações ambientais identificadas pelo grande desperdício de materiais descartados no processo de produção.

É certo que a Fabricação Digital trouxe mudanças significativas no processo de fabricação, viabilizando inovações e a execução de uma série de soluções antes inviáveis; entretanto, esse processo também desperta preocupações relacionadas ao meio ambiente. Nesse sentido, este artigo busca apresentar o impacto ambiental decorrente do uso dessa tecnologia, quando utilizado de forma aleatória bem como trazer uma reflexão sobre a importância de novos modos de projetar, incluindo a ética ambiental nesse processo.

\section{METODOLOGIA}

A pesquisa de mestrado completa será orientada por metodologia qualitativa, de caráter exploratório e deverá utilizar também o método da pesquisa-ação. De acordo com a definição por Thiollent (1988):

A pesquisa ação é um tipo de investigação social com base empírica que é concebida e realizada em estreita associação com uma ação ou com a resolução de um problema coletivo no qual os pesquisadores e os participantes representativos da situação ou do problema estão envolvidos de modo cooperativo ou participativo.

O processo da pesquisa seguirá três etapas fundamentais, combinando etapas teóricas e etapas práticas e incluem: 1) revisões bibliográficas relacionadas ao tema e estudos de casos; 2) estudos de campo em cursos de graduação (na UFU) que contém disciplinas relacionadas ao processo de projeto; 3) realização de workshops sobre fabricação digital e análises do processo de projeto e do projeto final; e 4) estruturação de diretrizes projetuais sustentáveis para a Fabricação digital para designers. 
Este artigo apresenta a primeira etapa da pesquisa, abordando questões relevantes sobre o processo de fabricação digital e pontuando reflexões sobre o impacto ambiental provocado pelo mesmo.

\section{REFLEXÕES A PARTIR DA FUNDAMENTAÇÃO TEÓRICA}

O procedimento de fabricação digital denominado file-to-factory (do arquivo para a fábrica) subtrai etapas de representações entre o projetista e o produto final (BARBOSA NETO et al., 2014). Neste cenário, o projetista está envolvido desde o processo de criação até a construção de uma forma (KOLAREVIC, 2005). Esse processo envolve a troca de informações entre softwares de modelagem tridimensional para uma máquina de fabricação digital, cujas fases estão baseadas em princípios computacionais (OOSTERHUIS, 2005). De acordo com Oxman (2006), a partir da FD, a informação passa a ser um "novo material" para o projetista.

Umas das principais características dessa tipologia de manufatura é a liberdade de criação, propiciando um alto nível de complexidade de projeto (Figura 1). Essa complexidade se realiza através dos softwares de modelagem baseados em NURBS (Non-Uniform Rational B-Splines), que viabilizam a criação de inúmeras curvas e superfícies paramétricas e o desenvolvimento de formas altamente complexas (PUPO, 2009).
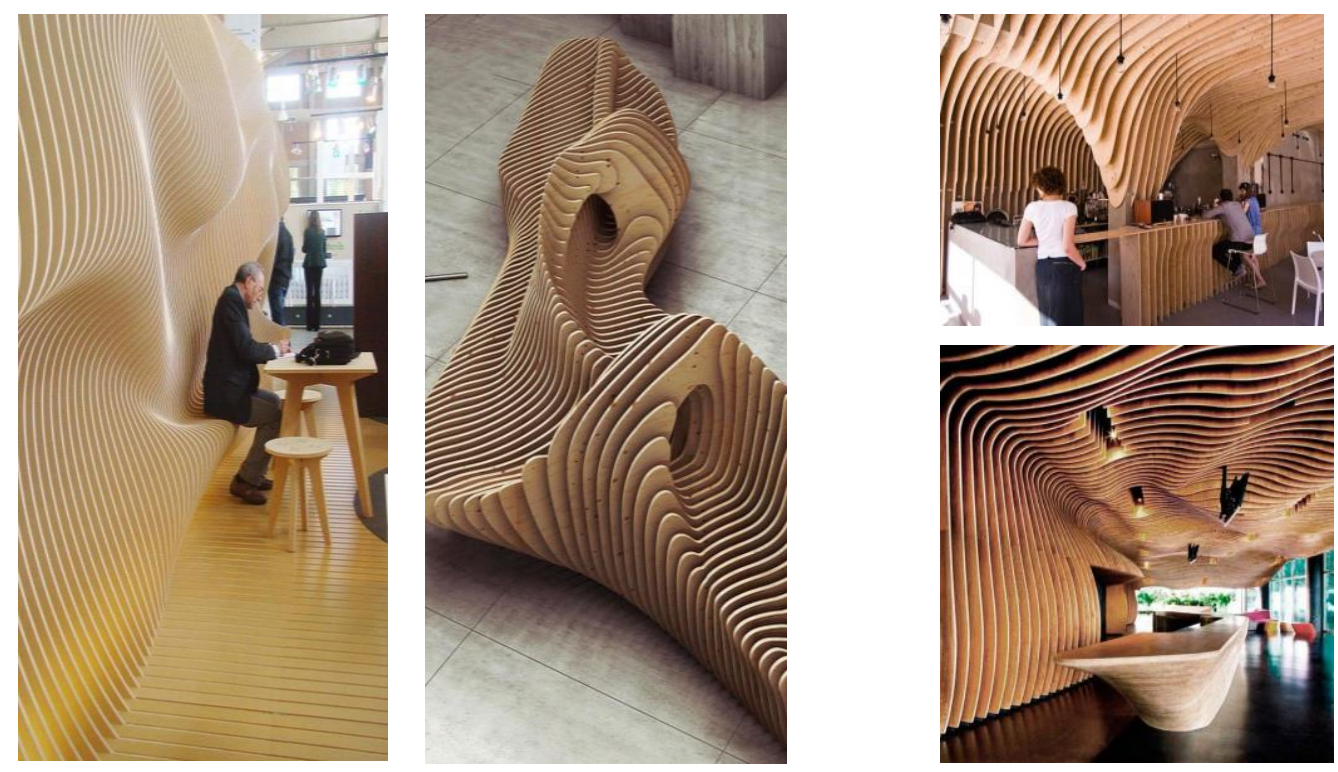

Figura 1 - Complexidade de Formas

Fonte: Pinterest (2019)

Apesar das grandes vantagens promovidas pela fabricação digital em suas aplicações, esta também tem despertado preocupações relacionadas ao meio ambiente.

Uma das questões está relacionada à facilidade de aquisição das máquinas de fabricação digital, o que demanda responsabilidade de uso dessas tecnologias. Tendo em vista que pessoas sem preparo técnico ou estudos em áreas de projeto podem usufruir das maquinas para produzir indiscriminadamente o que desejarem e, ainda, os vários sites disponíveis que fornecem projetos prontos gratuitos para a fabricação, denominados Opendesign ou Open-source (código aberto), o uso descontrolado e equivocado pode trazer sérias implicações ambientais.

De acordo com o autor Gershenfeld (2012), os projetos são desenvolvidos e 
compartilhados digitalmente, podendo ser produzidos em qualquer lugar do mundo. Por conseguinte, ocorre a descentralização dos meios de fabricação, tornando de livre acesso para todos os indivíduos.

Dando ênfase aos impactos ambientais, há uma grande preocupação em relação à fabricação de objetos que possuem geometrias complexas (característica da fabricação digital), em máquinas de manufatura subtrativa. Mesmo que ocorra o planejamento de corte antes da fabricação (por ex.: o uso de plug-in RhinoNest'l), quando os projetos são altamente complexos, contendo inúmeras curvas, o resultado pode gerar um grande volume de resíduos pequenos (Figura 2). Isso inviabiliza o reaproveitamento do material residual e, portanto, provoca sérios danos ambientais, tanto pelo volume de material inutilizado (ou desperdiçado) quanto pelo descarte incorreto, em muitos casos.

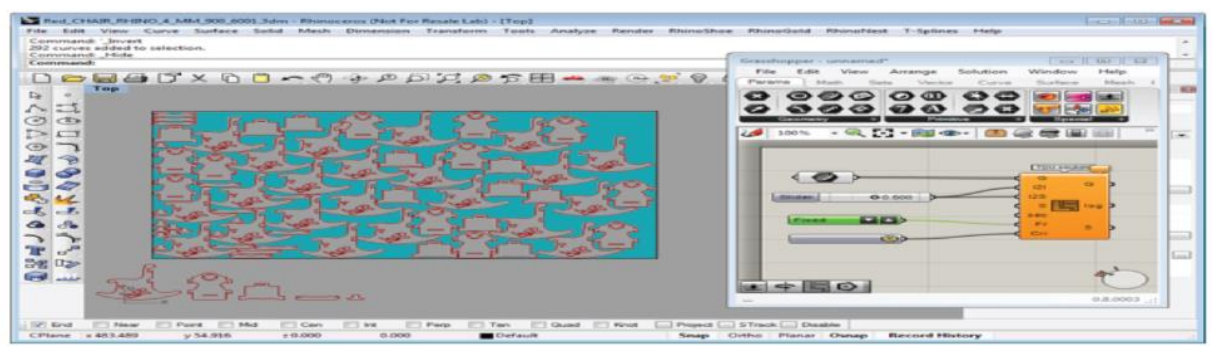

\section{Figura 2 - Planejamento de corte}

Fonte: Adaptado de: Disponível em:

http://help.tdmsolutions.com/rhinonest/3.0/en/index.html? Grasshopper.html (2018). Acesso em: 10 out. 2018

É possível observar que mesmo utilizando o planejamento de corte do plug-in RhinoNest, ainda sim há falhas. Conforme indicado na figura 3, o plug-in fornece um planejamento desorganizado das peças e, consequentemente, há um desperdício maior de resíduos pequenos. Utilizando as mesmas peças da figura anterior, figura 3 simula uma nova forma de organização das peças, com melhor aproveitamento da superfície da chapa.

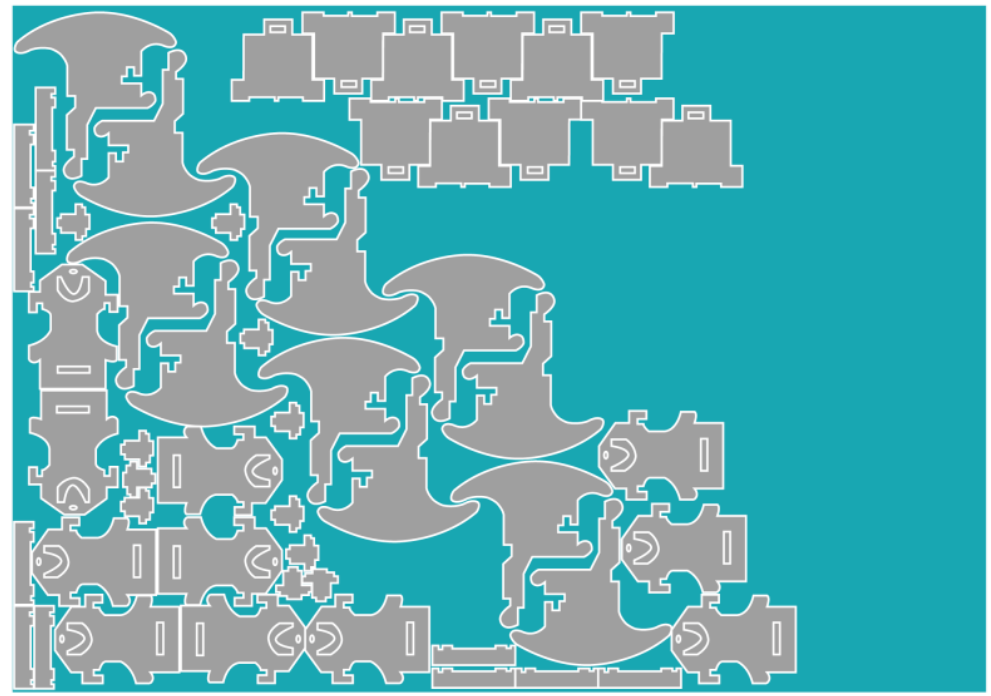

Figura 3 - Planejamento de corte adequado

Fonte: Autores (2018)

Embora ainda apresente perdas, em virtude da complexidade dos recortes, é possível observar uma redução significativa do volume de material descartado

\footnotetext{
${ }^{1}$ RhinoNest é um plug-in usado junto ao software de modelagem Rhino, que tem como função a geração do planejamento de corte.
} 
durante o processo de fabricação, quando o planejamento é programado de forma adequada. Nesse sentido, pode-se afirmar que o planejamento de corte é uma etapa de projeto fundamental para evitar desperdícios desnecessários de materiais, principalmente visando a esse modelo de projeto complexo possibilitado pela FD.

Segundo Walker (2005, p.3), a maioria dos produtos de consumo possui uma característica semelhante, "[...] a estética de um desperdício desmedido e de práticas social e ambientalmente danosas".

Não se pode negar que desenvolvimento dessas novas máquinas tecnológicas digitais contribui significativamente para o desenvolvimento em vários campos da sociedade; por outro lado, também impactam negativamente no espaço de trabalho, provocando riscos em função das demandas por produtividade cada vez maiores, tornando o ambiente exposto à certa deterioração de sua qualidade.

Em um mundo globalizado, o principal objetivo das empresas para mantê-las competitivas no mercado, é o ganho empresarial (FLORES; TERRIBILE, 2015). No entanto, "uma economia que não garante a conservação da vida em termos ambientais [...] não pode ser chamada de humana e, portanto, ética (VASCO, 2004 p.384)".

Diante disto, a visão da sociedade perante o meio ambiente não pode ser exclusivamente econômica, ou seja, tem que ser direcionada também pela perspectiva ética (LOURENÇO, 2012 apud FLORES; TERRIBILE, 2015). Essa mudança parte não apenas da visão das empresas, mas deve incluir a busca por uma mudança de postura comportamental, alterando o modo de pensar e agir configurando nossos valores (CAPRA, 2006).

De acordo com o autor Vasco (2004), a economia em equilíbrio com a ética materializa uma economia real, isto é, uma economia direcionada pelos princípios éticos que promovem as demandas da vida global e asseguram a progresso desta. Para Flores e Terribile (2015), essa mudança de postura só será possível a partir da adoção da "ética ocupacional sustentável" que prevê privilegiar o meio ambiente nos sistemas de produção e consumo.

Segundo Manzini (2008)

\begin{abstract}
A transição rumo à sustentabilidade será um processo de aprendizagem social no qual os seres humanos aprenderão gradualmente, através de erros e contradições [...], a viver melhor consumindo (muito) menos e regenerando a qualidade do ambiente, ou seja, do ecossistema global e dos contextos locais ondem vivem (MANZINI, 2008, p.27).
\end{abstract}

Desde sua origem, o termo "ético" faz referência à sociedade como um todo, e não apenas referente ao indivíduo, sendo considerada, assim, uma atividade social com comunicação entre os indivíduos. A ética refere-se à conexão entre a realidade e a ações humanas. Esta última está associada ao conhecimento (percepção da realidade) e à ação (desejo do indivíduo sobre o entendimento, adaptação e transformação) perante a realidade (VASCO, 2004).

Para tanto, o primeiro passo é identificar o problema e fazer uma reflexão em relação ao nosso papel e esforço de colaboração. Uma questão relevante seria iniciar essa reflexão relacionada à atuação do designer e à estética do projeto, ponderando a correlação da estética e da prática de fabricação insustentável (WALKER, 2005). 
[...] Novos processos produtivos requer a visualização do homem como um elemento/parte integrante da sustentabilidade profunda (p.12). [...] (FLORES; TERRIBILE, 2015).

A inserção da ética é, portanto, de extrema importância nas relações humanas, principalmente em pesquisas e ações ambientais que possuem como objetivo a recuperação e preservação do meio ambiente. Isso significa não somente seguir regulamentos, mas também projetar soluções e programas ambientais sustentáveis, aplicados à todas as atividades e iniciativas da sociedade, em busca da "máxima definição da sustentabilidade" (HENKES, 2016).

De acordo com Vasco (2004), quando se definem e aplicam-se ações referentes a um contexto da sociedade, tal ação é considerada parte da ética. Neste contexto, todo processo de projeto tem que ser guiado por determinações éticas sustentáveis para garantir a sobrevivência da sociedade em um mundo protegido ambientalmente.

Levando em consideração estas questões, a pesquisa, como um todo, visará ao desenvolvimento de diretrizes projetuais para a Fabricação digital para minimizar os resíduos gerados na fabricação subtrativa. Entende-se que essas estratégias são de extrema importância para diminuir o impacto ambiental que os processos de fabricação em geral e os produtos finais vêm causando, em busca de soluções mais sustentáveis.

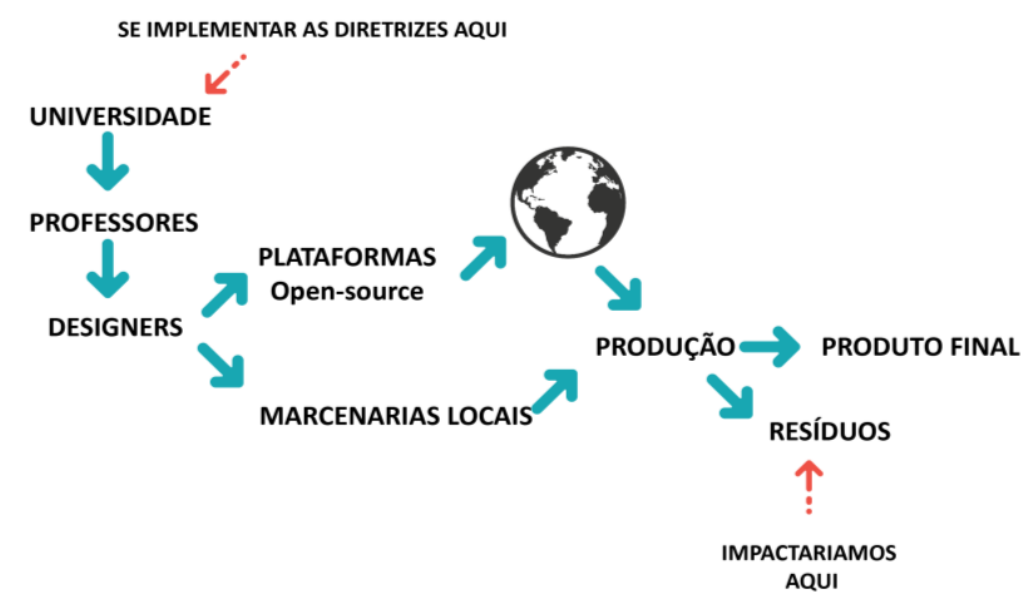

Figura 4 - Esquema de implementação das diretrizes

Fonte: Autores (2018)

A implementação de diretrizes sustentáveis (Figura 3) no ensino de projeto nos cursos de graduação em design poderá resultar em um novo modo de projetar. Além disso, poderá contribuir para o surgimento de uma nova configuração de produtos disponibilizados em plataformas abertas, impactando positivamente para a redução de resíduos de projetos fabricados em qualquer lugar do mundo.

A preocupação relativa às condições ambientais do planeta, em busca da manutenção do seu equilíbrio, possibilita orientar um melhor futuro para a humanidade, a partir da evolução do conhecimento ético-ambiental para as próximas gerações (HENKES, 2016).

\section{CONSIDERAÇÕES FINAIS}

Tendo em vista as questões apresentadas, especialmente considerando os limites de recursos naturais do planeta, o uso das manufaturas da fabricação 
digital deve ser mais rigoroso e demanda um compromisso de 'projetar responsável', antevendo e evitando suas implicações ambientais negativas.

A partir das pesquisas realizadas até o momento, observa-se que o baixo desempenho dos plug-ins de planejamento de corte dos softwares de modelagem tem contribuído para a geração de resíduos pequenos na etapa de fabricação.

Nesse sentido, as estratégias de projeto mencionadas devem, portanto, buscar - fundamental equilíbrio entre a liberdade de criação e o processo de fabricação, sendo orientadas pela responsabilidade e ética projetual relacionadas ao meio ambiente, em busca do aprendizado social que deve ser intrínseco a todos os indivíduos, principalmente aos projetistas.

Destaca-se o importante papel do designer nesse cenário, com a urgente necessidade de se posicionar e buscar uma mudança radical no processo de desenvolvimento de novos produtos, adotando medidas sustentáveis, e inserindo a "estética verde" como uma nova configuração de produtos.

Entretanto, essa mudança de postura não é direcionada apenas aos designers. Considerando-se a facilidade de acesso aos projetos disponibilizados em plataformas digitais, e executados por pessoas "leigas", é fundamental que esses indivíduos também estejam conscientes e criticamente atentos com as escolhas de projeto e seus possíveis impactos. Isso poderá estimular a adoção de critérios ambientais que contribuam para direcionar a seleção de um projeto menos impactante ambientalmente.

Por fim, é indispensável aprimorar a etapa de planejamento de corte em tecnologias de fabricação digital por subtração afim de minimizar, ao máximo possível, o desperdício desnecessário de materiais. Todas as estratégias, adotadas em conjunto, serão de extrema importância para se encontrar soluções mais sustentáveis e se atingir um estágio mais eficiente de produção, reduzindo os enormes impactos ambientais que os processos produtivos, na sua maioria, vêm causando ao planeta.

\section{REFERÊNCIAS}

BARBOSA NETO, W.et al. Samba reception desk: Compromising aesthetics, fabrication and structural performance with the use of virtual and physical models in the design process. Gestão e Tecnologia de Projetos, São Paulo, v. 9, n. 2, p.53-69, jul./dez. 2014. Disponível em:

<http://dx.doi.org/10.11606/gtp.v9il.83913>

CAPRA, Fritjof. A TEIA DA VIDA - Uma nova compreensão científica

dos sistemas vivos. Tradução: Newton Roberval Eichemberg. Editora Cultrix: São Paulo, 2006.

FLORES, Nilton Cesar; TERRIBILE, Daniele Regina. Ética ocupacional sustentável numa sociedade globalizada. Revista do Direito Público, [s.l.], v. 10, n. 2, p.89110, 1 set. 2015. Universidade Estadual de Londrina. Disponível em: <http://dx.doi.org/10.5433/1980-51 1x.2015v10n2p89>.

GERSHENFELD, Neil. How to Make Almost Anything: The Digital Fabrication

Revolution. Foreign Affairs, [s. I.], v. 91, n. November/December, 2012. Disponível em: <http://cba.mit.edu/docs/papers/12.09.FA.pdf>

HENKES, Jairo Afonso. ÉTICA AMBIENTAL E TECNOLOGIAS APLICÁVEIS AO

DESENVOLVIMENTO SUSTENTÁVEL. Gestão Sustentável Ambiental, Florianópolis, V. 5, n. 1, p.1-4, 2016. 
HOPKINSON, N.; HAGUE, R.J.M.; DICKENS, P.M. Rapid Manufacturing an Industrial Revolution for the digital age. Wiley, Chichester, 2006.

KOLAREVIC, Branko (Ed.). Architecture in the digital age: design and manufacturing. New York: Taylor e Francis, 2005. 314 p.

MANZINI, EZio. Design para a inovação social e sustentabilidade: Comunidades criativas, organizações colaborativas e novas redes projetuais. Tradução de Carla Cipolla. Rio de Janeiro: E-papers, 2008. 103 p.

MENICHINELLI, Massimo. A data-driven approach for understanding Open Design. Mapping social interactions in collaborative processes on GitHub, The Design Journal, 20:sup 1. (2017) , S3643-S3658,

DOI:10.1080/14606925.2017.1352869

OOSTERHUIS, K. File to Factory and Real Time Behavior in ONL-Architecture. 2005. Disponível em: <http://papers.cumincad.org/data/works/att/acadia 04_294.content.pdf> Acesso em: 20 outubro 2018.

OXMAN, R. Theory and design in the first digital age. Design Studies, v. 27, n.3, p.229-265, 2006. http://dx.doi.org/10.1016/i.destud. 2005.11.002PINTEREST. 2019. Disponível em: <https://br.pinterest.com/>. Acesso em: 10 jan. 2019.

PUPO, Regiane Trevisan. Inserção da PROTOTIPAGEM e FABRICAÇÃO DIGITAIS no processo de projeto: um novo desafio para o ensino de arquitetura. 2009. 259 f. Tese (Doutorado) - Curso de Engenharia Civil, Universidade Estadual de Campinas, Campinas, 2009.

THIOLLENT, Michel. Metodologia da Pesquisa-Ação. São Paulo: Cortez,1985. VASCO, Luis Augusto Panchi. DE ÉtICA ECONÓMICA A ECONOMÍA Y ÉTICA: FUNDAMENTOS A PARTIR DE UNA RACIONALIDAD ÉTICO INTERPRETATIVA, CON UNA APLICACIÓN AL CASO ECUATORIANO. Quito: Abya-yala, 2004. 481 p.

WALKER, Stuart. Desmascarando o objeto: reestruturando o design para sustentabilidade. Revista Design em Foco, Salvador, v. 2, n. 2, p.47-62, 2 jul. 2005. Trimestral. 